\title{
Contribution of globular clusters to halos
}

\author{
Angela Bragaglia \\ INAF-Osservatorio Astronomico di Bologna, via Ranzani 1, 40127 Bologna, Italy \\ email: angela.bragaglia@oabo.inaf.it
}

\begin{abstract}
The contribution of massive star clusters to their hosting halo dramatically depends on their formation mechanism and their early evolution. Massive globular clusters in the Milky Way (and in other galaxies) have been shown to display peculiar chemical patterns (lightelements correlations and anti-correlations) indicative of a complex star formation, confirmed by photometric evidence (spread or split sequences). I use these chemical signatures to try to understand what is the fraction of halo stars originally born in globular clusters.
\end{abstract}

Keywords. Galaxy, halo, globular clusters

\section{Introduction}

Globular cluster (GC) systems are found in all kind of galaxies, although the number and frequency of GCs may vary a lot, depending also on the morphological type (e.g., Brodie \& Strader 2006). In particular, in the Milky Way (MW) the specific frequency, that is the number of clusters per unit galaxy luminosity, is low, as typical of spirals (see, e.g. the recent work by Harris et al. 2013).

The MW hosts about 160 GCs, two-thirds of which reside in the halo (e.g. Harris 1996 and web updates). Possibly this number will increase a lot, since new additions from various surveys such as SDSS, Pan-STARRS, DES, VST ATLAS, etc are nowadays common, see e.g., Koposov et al. (2007), Laevens et al. (2015), Luque et al. (2015), Belokurov et al. (2014), even if the objects found are sometimes classified as GCs or ultrafaint dwarfs depending on the study, as is the case of the last example given here. However, these new clusters are all rather small, we do not expect to have missed really massive clusters in the halo.

Clusters lose mass and stars during their evolution (see, e.g., Baumgardt et al. 2008). This is due to several effects, first the violent relaxation phase and later two-body encounters and tidal shocks with disk and bulge, on longer timescales. Furthermore, some clusters have a combination of mass and size which put them outside the survival zone (defined in Gnedin \& Ostriker 1997), see for instance Jordi \& Grebel (2010). The less massive clusters may even be completely dissolved, so that the GCs we see today are the remnants of a larger population and are less massive than at birth, having contributed, and still contributing, stars to the MW halo. One very famous case is Pal 5, with its very extended double tidal tail, discovered by Odenkirchen et al. (2001). Many other tails have been found in the halo, some still connected with a cluster (e.g., NGC 288, Grillmair et al. 2013 or Pal 14, Sollima et al. 2011), but many more without a clearly associated progenitor cluster. For instance, Grillmair (2015) counted 21 such streams and calculated that the original GC number should have been about 450 . These streams and tails are direct proofs that clusters contribute(d) to the halo. However, it is not yet clear how large the contribution is. 


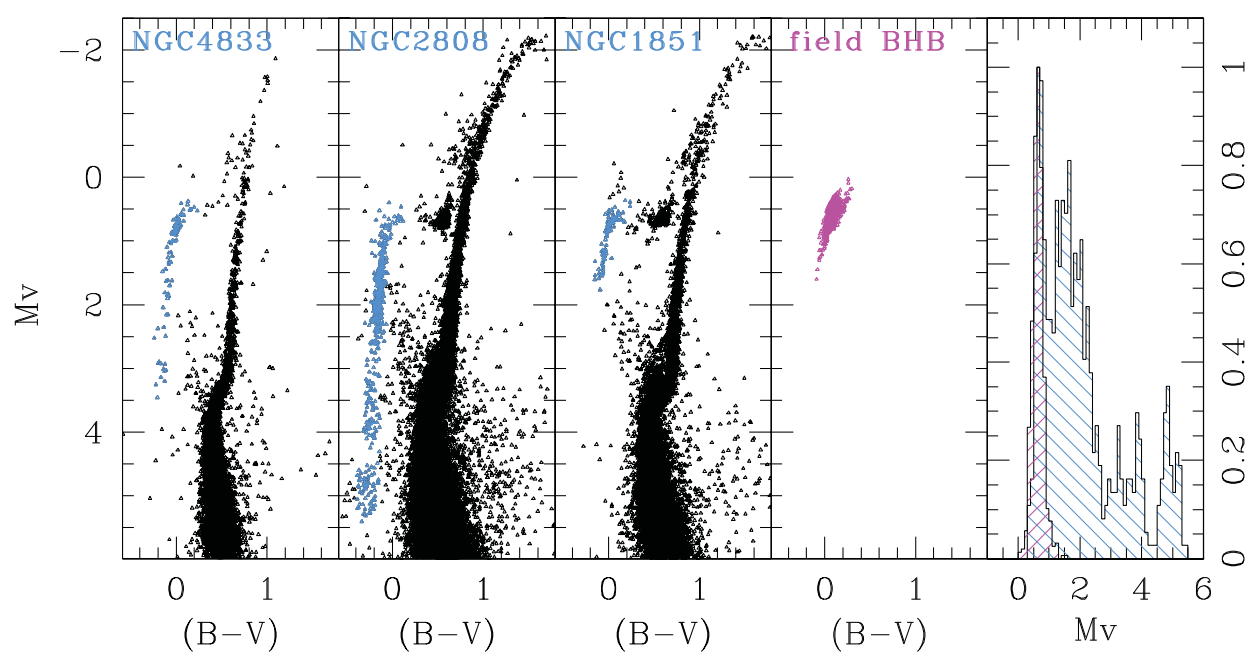

Figure 1. Three CMDs corrected for reddening of GCs (photometry taken from Piotto et al. 2002) and CMD for field BHB (Brown et al. 2008); in the four panels the BHBs are indicated by coloured points. Right panel: histogram of the absolute magnitudes of field BHBs (in magenta, very peaked, for about 650 stars) and the three GCs (in blue, more extended, for about 630 stars).

\section{Field halo and GC stars: is chemical tagging possible?}

Globular cluster and field halo stars share in fact many properties. For instance, they have very similar metallicity distributions (see e.g., Fig. 4 in Gratton et al. 2012), peaked near $[\mathrm{Fe} / \mathrm{H}] \simeq-1.5$. They are also indistinguishable when their $\alpha$-elements are considered (see e.g., Fig. 1 in Carretta 2015), while the same is not not true for other components, like the MW satellites, which generally show lower $[\alpha / \mathrm{Fe}]$ ratios.

However, GCs and halo field stars do also show striking differences, for instance, the horizontal branch morphology. The absolute magnitude and the colour of the blue horizontal branch (BHB) stars show very dissimilar distributions. This is shown in Fig. 1, where the colour-magnitude diagrams (CMD) of three GCs, and in particular their BHBs, are compared to field BHBs. The colour and luminosity distributions are clearly not identical; in particular, the GCs seem to possess an additional component, hotter and possibly enriched in helium (e.g., D'Antona \& Caloi 2004).

This is most probably connected with the peculiar chemistry shown by GC stars for light elements (C, N, O, Mg, Al, Na). For instance, it has long been known than carbon and nitrogen have large spreads anticorrelated with each other and also show bimodality (e.g., Norris et al. 1984, Cohen et al. 2002, Pancino et al. 2010). Furthermore, GCs show large star-to-star variations in sodium and oxygen (and sometimes in aluminum and magnesium), anticorrelated with each other, which are not present among field stars (see e.g. the reviews in Kraft 1994, Gratton et al. 2004, Gratton et al. 2012), at least not generally, as we will see next. These (anti)correlations are the signature of hydrogen burning at high temperature; the current understanding is that they are due to a previous generation of stars, more massive than the ones presently alive in GCs, which polluted the material from which (a) second generation(s) of stars formed (as discussed by many in this Symposium, e.g., Charbonnel in the plenary talk, Marino - and Bastian for a distinctive view). These generations will be addressed as first generation (FG) and second generation (SG) in the following. 


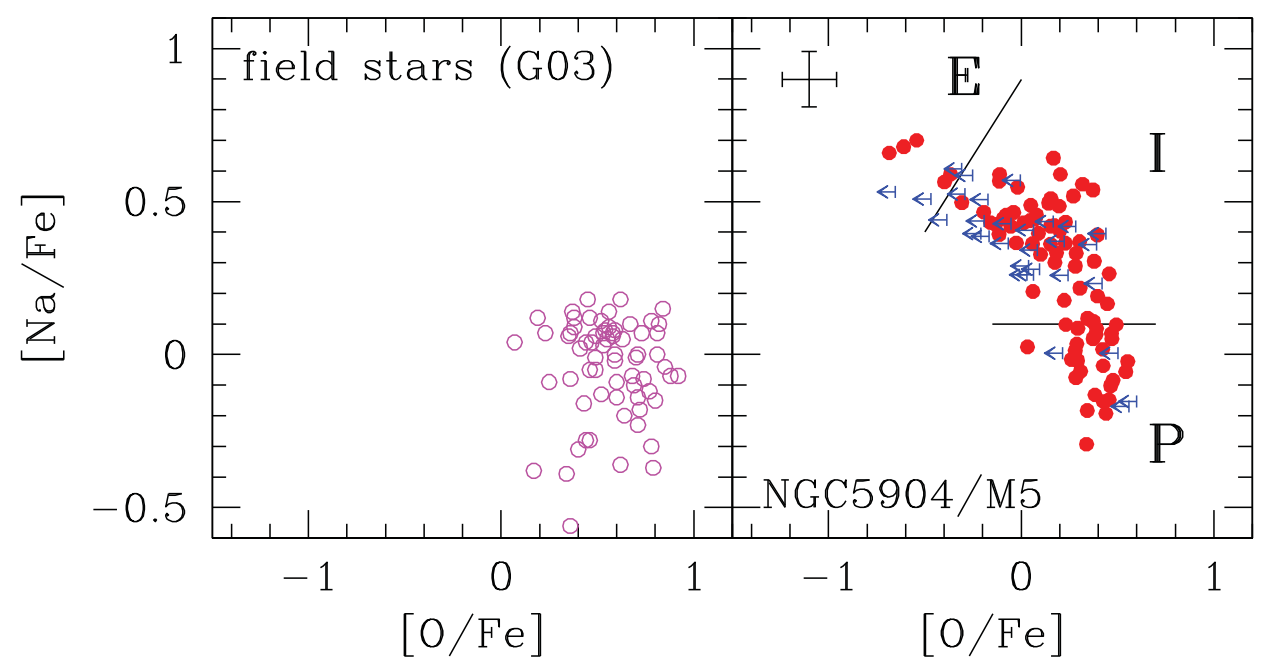

Figure 2. $[\mathrm{O} / \mathrm{Fe}]$ vs $[\mathrm{Na} / \mathrm{Fe}]$ for field stars (left, data from Gratton et al. 2003) and a typical GC, M5 (right, data from Carretta et al. 2009a,b). For the GC the separation between FG (here indicated by $\mathrm{P}$, which stands for primordial coposition) and SG stars (indicated by I, E, standing for intermediate and extreme composition) is also presented.

This behaviour is displayed in Fig. 2 for $\mathrm{Na}$ and $\mathrm{O}$ : the metal-poor field halo stars occupy only a narrow region in the O-Na plane, while the GC stars have an additional large dispersion in both elements. This is shown in Fig. 2 for a single GC, but the same behaviour is seen in practically all the MW GCs massive enough, as demonstrated by many studies summarized in the review papers cited just above, from the small Pal 5 (Smith et al. 2002) to the huge $\omega$ Cen (e.g. Johnson \& Pilachowski 2010, Marino et al. 2011). In particular, it is seen in the large, homogeneous survey of red giant stars in 26 MW GCs done with FLAMES@VLT and presented in a series of papers (e.g., Carretta et al. 2006 and Bragaglia et al. 2015 to cite only the first and the latest). Carretta et al. (2010) discussed the "universality" of the Na-O anticorrelation among massive clusters, proposing it as a defining property of globulars.

Adopting the mainstream interpretation, in each GC there are two components, one of primordial composition (the FG) and one with modified composition (the SG). Interestingly, the fraction of FG to SG stars in clusters is remarkably constant. Carretta et al. (2010) and following papers of the FLAMES series found about one third and two thirds of FG and SG stars, respectively. This has recently been confirmed by Bastian \& Lardo (2015), who added a few more clusters from other sources, but also noted that the almost constant fraction of SG to FG stars is not in ageement with models of mass loss from clusters.

As it has been remarked by many studies, this large fraction of SG stars leads to a mass-budget problem. If they formed from the ejecta of FG stars, only a small part of the original stellar cluster mass went into the SG. This seems to imply than the clusters were originally much more massive, even tens of times more massive than today (and/or that the FG had a peculiar initial mass function, as discussed by others in this Symposium. e.g., Charbonnel, Larsen). If clusters were only slightly more massive than today, then the halo contains only a few per cent of stars lost by GCs. But if clusters lost more than $90 \%$ of their original stars, as advocated by many models of GC formation in the context of multiple generations, the halo contains instead a significant fraction of stars born in GCs (the exact number depends on the model adopted for FG polluting stars, 


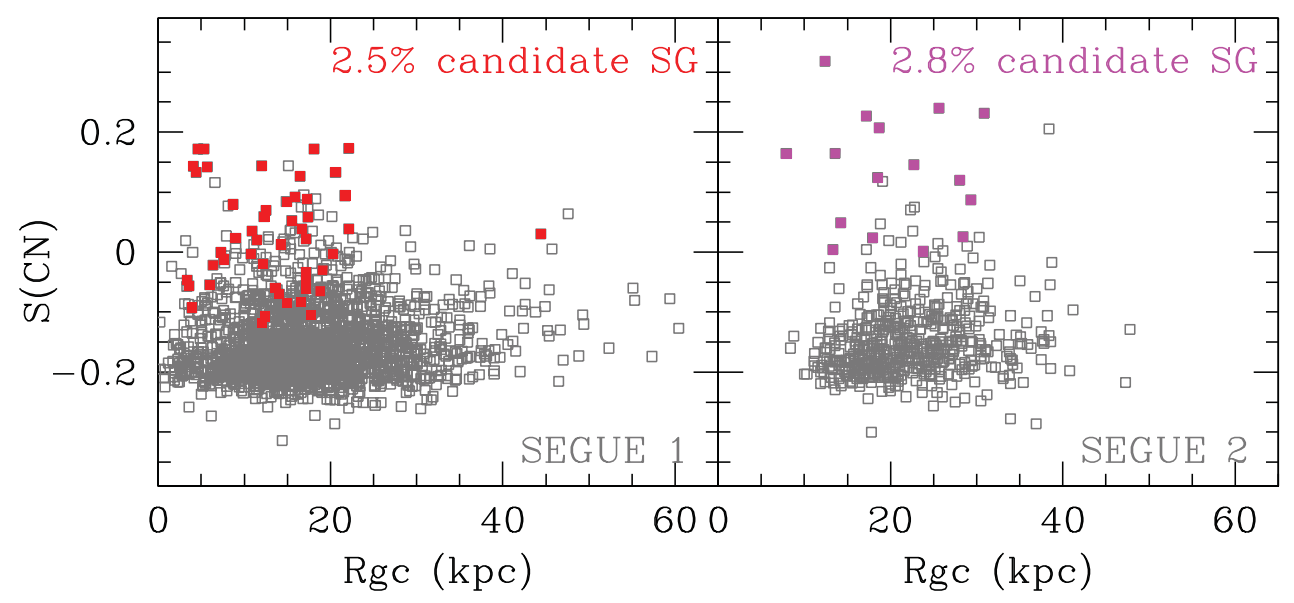

Figure 3. CN enhancement in SEGUE 1 and SEGUE 2 stars, from Martell \& Grebel (2010) and Martell et al. (2011). Filled, coloured symbols indicate candidate SG-like stars. Note that $\delta \mathrm{S}(\mathrm{CN})$ is used in the original papers, while here $\mathrm{S}(\mathrm{CN})$ is plotted.

see e.g., Gratton et al. 2012 for a discussion). Especially in the second case, the stars lost were almost all of FG, the ones that have a composition similar to the field halo stars. This means that it is very difficult, if not impossible, to distinguish them using chemical tagging.

\section{GC stars: lost and found}

On the other hand, it seems rather "easy" to find SG-like stars, given their peculiar chemistry (enhancement in $\mathrm{N}, \mathrm{Na}$, and $\mathrm{Al}$, combined with depletion of $\mathrm{C}, \mathrm{O}$, and $\mathrm{Mg}$ in the same star).

Martell \& Grebel (2010) and Martell et al. (2011) focused on C and N, measuring the $\mathrm{CN}$ and $\mathrm{CH}$ band strengths on the low resolution spectra of about 2500 giant stars in the SEGUE 1 and SEGUE 2 samples. They found 49 CN-strong (i.e., SG-like) stars over 1957 and 16 over 561 in the two samples, respectively. Figure 3 is built using data from their papers; in both cases the observed fraction is nearly $3 \%$.

If we consider instead $\mathrm{Na}$ and $\mathrm{O}$, we are aware of two different instances. Carretta et al. (2010) considered the minimum and maximum $\mathrm{Na}$ abundance in their sample of nearly 20 GCs, that are indicative of FG and SG stars respectively, and compared them with the $\mathrm{Na}$ abundance of field halo stars of similar metallicity. After discarding 4 stars with high $\mathrm{Na}$ abundance because their overabundance was most probably due to a companion AGB star, they were left with 2 stars out of 144 , deducing a fraction of $1.4 \%$ SG-like stars. Considering the present-day fraction of SG and FG stars, this means a minimum observed GC contribution to the halo of about 3\%. A similar fraction was also found by Ramírez et al. (2012), who discovered 2 Na-rich and O-poor stars while studying 67 nearby halo stars.

Another possibility for chemical tagging halo stars comes from the $\mathrm{Mg}$-Al anticorrelation. It is not seen in all clusters (see Carretta et al. 2009b), but Al can show even huge variations between FG and SG stars, more than 1 dex, so that in principle it is easy to find stars with these extreme chemical properties. Figure 4 (left panel) shows the case for NGC 6752, where Carretta et al. (2012) found a very extended distribution in $\mathrm{Mg}$ and especially Al. This feature was exploited by Lind et al. (2015); they employed the first data release of the large public Gaia-ESO spectroscopic survey (Gilmore et al. 2012, 


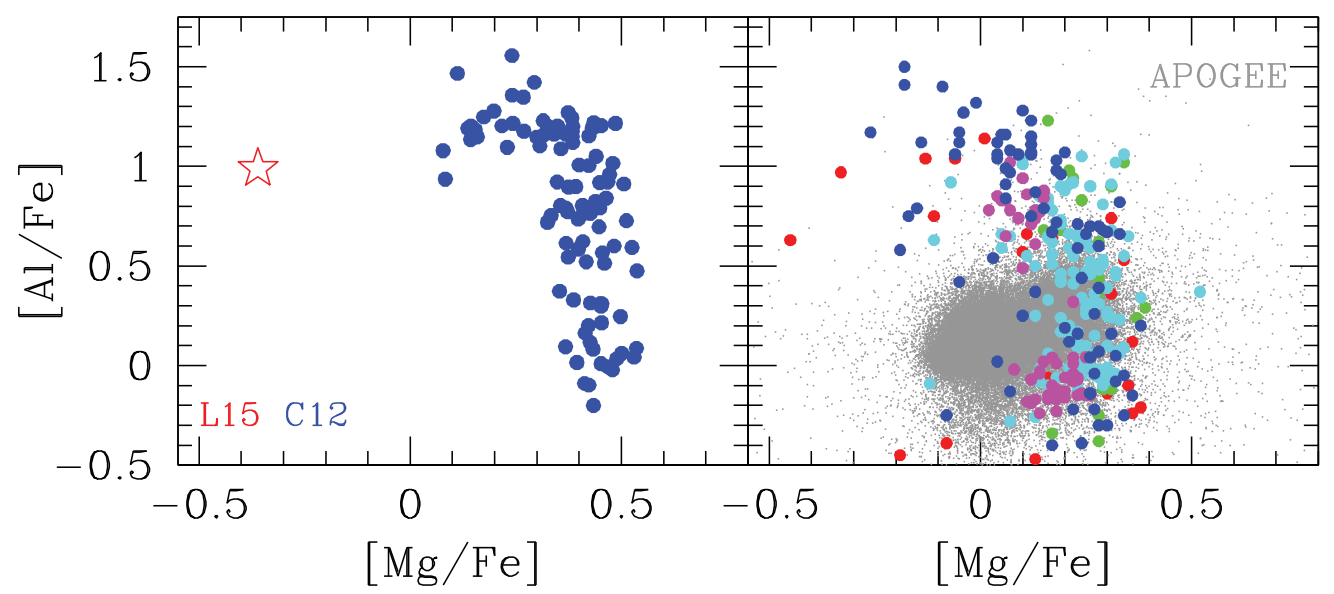

Figure 4. Left panel: $[\mathrm{Mg} / \mathrm{Fe}]$ vs $[\mathrm{Al} / \mathrm{Fe}]$ for NGC 6752 (blue filled circles, from Carretta et al. 2012) and one candidate escapee from a GC (red star, from Lind et al. 2015. Right panel: APOGEE data are used here; gray dots are field stars, larger, coloured symbols are stars in five GCs (M2, M3, M5, M13, M15) from Mészáros et al. (2015).

Randich et al. 2013) to find a good candidate escapee from a GC, shown in Fig. 4 as a star symbol. Unfortunately, the Gaia-ESO setup for the bulk of field MW stars does not cover lines of sodium and oxygen. More candidates will probably be found in more advanced releases and in the final catalogue, so that it will also be possible to do some counts on fractions and calculate the GC contribution to the halo.

The same kind of search could be done also on data from other surveys, for instance APOGEE. The right panel of Fig. 4 shows the $\mathrm{Mg}$ and $\mathrm{Al}$ abundances taken from the latest data release and the values for a few GCs (Mészáros et al. 2015); some of the stars seem to reach the high $\mathrm{Al}$ values we are looking for in SG-like stars.

This chemical tagging with connected enhancements and depletions in light elements is being used also to confirm the associations of streams and moving groups with clusters. Examples of a positive and negative identification, respectively, are the Aquarius stream (consistent with being GC debris, Wylie-de Boer et al. 2012) and the Kapteyn group (not associated with $\omega$ Cen, Navarrete et al. 2015).

Finally, I wish to mention one interesting finding that could indicate a connection between halos and GCs. Greene et al. (2015), Greene (2015), in their MASSIVE survey of early-type galaxies, found that nitrogen abundance does not decline outwards as iron and other elements do. Apparently the outer regions of these galaxies, which we may associate with the halo, are rich in nitrogen, as SG stars are in GCs; it may be just a concidence, or it may have other explanations, but it is intriguing.

\section{Summary}

There are theoretical reasons to expect that GCs contribute(d) part (and even most, depending on the proposed scenarios fo multiple stellar populations in GCs) of their stars to the MW field halo. However, using only models of cluster formation and disruption/mass loss, it is not currently assessed how important this contribution is.

On the observational side, there is evidence of disrupting and disrupted clusters in the form of tidal tails, streams, and moving groups. Furthermore, we may use chemical tagging to find stars, presently in the MW halo field, which were originally born in a GC. The halo field stars and the GCs share many chemical properties, but also some 
striking differences: GC stars show a large star-to-star dispersion in light elements, which is considered proof of the existence of multiple populations in clusters. This is not visible in the bulk of field halo stars.

We may then try to find out halo stars with similar fingerprints, looking in particular to elements like nitrogen, sodium, and aluminum, which show large enhancement in SG GC stars (anticorrelated with carbon, oxygen, and magnesium, respectively). This has been done by a few studies (Carretta et al. 2010, Martell et al. 2011, Ramirez et al. 2012, and Lind et al. 2015) and can be done on a larger scale using the large ground-based spectroscopic surveys, like APOGEE and Gaia-ESO.

Using the numbers of observed SG-like stars in the MW halo (about 1.5 to $3 \%$ ), and bearing in mind that present-day GCs host about 70\% SG and 30\% FG stars, we may deduce that the minimum contribution of GCs is about 5\%. This fraction might increase even a lot if clusters were really much more massive at their birth as advocated by most models trying to explain the multiple populations in GCs. However, the subject is still hotly debated and we need improved models and more observations.

I acknowledge the financial support of IAU and of the Italian MIUR (PRIN "The Chemical and Dynamical Evolution of the Milky Way and Local Group Galaxies", PI F. Matteucci).

\section{References}

Bastian, N. \& Lardo, C. 2015, MNRAS, 453, 357

Baumgardt, H., Kroupa, P., \& Parmentier, G. 2008, MNRAS, 384, 1231

Belokurov, V., Irwin, M. J., Koposov, S. E., et al. 2014, MNRAS, 441, 2124

Bragaglia, A., Carretta, E., Sollima, A., et al. 2015, A\&SA (in press), arXiv:1507.07562

Brodie, J. P. \& Strader, J. 2006, ARA\& A, 44, 193

Brown, W. R., Beers, T. C., Wilhelm, R., et al., 2008, AJ, 135, 564

Carretta, E., Bragaglia, A., Gratton, R. G., et al. 2006, A\&A, 450, 523

Carretta, E., Bragaglia, A., Gratton, R. G., et al. 2009a, A\&A, 505, 117

Carretta, E., Bragaglia, A., Gratton, R., \& Lucatello, S. 2009b, A\&A, 505, 139

Carretta, E., Bragaglia, A., Gratton, R. G., et al. 2010, A\& A, 516, A55

Carretta, E., Bragaglia, A., Gratton, R. G., Lucatello, S., \& D'Orazi, V. 2012, ApJ (Letters), $750, \mathrm{~L} 14$

Carretta, E. 2015, IAUS 317, IAU General Assembly \#29, \#2252181, arXiv:1510.00507

Cohen, J. G., Briley, M. M., \& Stetson, P. B. 2002, AJ, 123, 2525

D'Antona, F. \& Caloi, V. 2004, ApJ, 611, 871

Gilmore, G., Randich, S., Asplund, M., et al. 2012, The Messenger, 147, 25

Gnedin, O. Y. \& Ostriker, J. P. 1997, ApJ, 474, 223

Gratton, R. G., Carretta, E., Desidera, S., et al. 2003, A\&A, 406, 131

Gratton, R., Sneden, C., \& Carretta, E. 2004, ARA\&A, 42, 385

Gratton, R. G., Carretta, E., \& Bragaglia, A. 2012, A\&ARv, 20, 50

Greene, J. 2015, IAUS 317, IAU General Assembly \#29, \#2250981

Greene, J. E., Janish, R., Ma, C.-P., et al. 2015, ApJ, 807, 11

Grillmair, C. J., Cutri, R., Masci, F. J., et al. 2013, ApJ (Letters), 769, L23

Grillmair, C. J. 2015, IAUS 317, IAU General Assembly \#29, \#2255751

Harris, W. E. 1996, AJ, 112, 1487

Harris, W. E., Harris, G. L. H., \& Alessi, M. 2013, ApJ, 772, 82

Johnson, C. I. \& Pilachowski, C. A. 2010, ApJ, 722, 1373

Jordi, K. \& Grebel, E. K. 2010, $A \& A$, 522, A71

Koposov, S., de Jong, J. T. A., Belokurov, V., et al. 2007, ApJ, 669, 337

Kraft, R. P. 1994, PASP, 106, 553 
Laevens, B. P. M., Martin, N. F., Bernard, E. J., et al. 2015, arXiv:1507.07564

Lind, K., Koposov, S. E., Battistini, C., et al. 2015, A\&A, 575, L12

Luque, E., Queiroz, A., Santiago, B., et al. 2015, arXiv:1508.02381

Marino, A. F., Milone, A. P., Piotto, G., et al. 2011, ApJ, 731, 64

Martell, S. L. \& Grebel, E. K. 2010, A\& A, 519, A14

Martell, S. L., Smolinski, J. P., Beers, T. C., \& Grebel, E. K. 2011, A\&\&A, 534, A136

Mészáros, S., Martell, S. L., Shetrone, M., et al. 2015, AJ, 149, 153

Navarrete, C., Chanamé, J., Ramírez, I., et al. 2015, ApJ, 808, 103

Norris, J., Freeman, K. C., \& Da Costa, G. S. 1984, ApJ, 277, 615

Odenkirchen, M., Grebel, E. K., Rockosi, C. M., et al. 2001, ApJ (Letters), 548, L165

Pancino, E., Rejkuba, M., Zoccali, M., \& Carrera, R. 2010, A\& A, 524, A44

Piotto, G., King, I. R., Djorgovski, S. G, et al., 2002, A\& $A$, 391, 945

Ramírez, I., Meléndez, J., \& Chanamé, J. 2012, ApJ, 757, 164

Randich, S. \& Gilmore, G., Gaia-ESO Consortium 2013, The Messenger, 154, 47

Smith, G. H., Sneden, C., \& Kraft, R. P. 2002, AJ, 123, 1502

Sollima, A., Martínez-Delgado, D., Valls-Gabaud, D., \& Peñarrubia, J. 2011, ApJ, 726, 47

Wylie-de Boer, E., Freeman, K., Williams, M., et al. 2012, ApJ, 755, 35 